\title{
Publisher's Note: Estimation of the diffusion constant from intermittent trajectories with variable position uncertainties [Phys. Rev. E 93, 042401 (2016)]
}

Peter K. Relich, Mark J. Olah, Patrick J. Cutler, and Keith A. Lidke

(Received 13 April 2016; published 25 April 2016)

DOI: 10.1103/PhysRevE.93.049906

This paper was published online on 4 April 2016 with an error in Eq. (27) and the equation above it. The equation above Eq. (27) should read as

$$
P(\ln D \leqslant A) \approx \int_{-\infty}^{A} d(\ln D) \mathcal{N}\left(\ln D, \ln \hat{D}, \tilde{\mathcal{K}}(\ln \hat{D})^{-1}\right)=\frac{1}{2}\left[1+\operatorname{erf}\left(\frac{A-\ln \hat{D}}{\sqrt{2 \tilde{\mathcal{K}}(\ln \hat{D})^{-1}}}\right)\right] .
$$

Equation (27) should read as

$$
B_{ \pm}=\exp \left[\ln \hat{D} \pm \sqrt{2 \tilde{\mathcal{K}}(\ln \hat{D})^{-1}} \operatorname{erf}^{-1}(C)\right]
$$

The equations have been corrected as of 13 April 2016. The equations are correct in the printed version of the journal. 